\title{
Chemotherapy-induced alopecia management: Clinical experience and practical advice
}

\author{
Alfredo Rossi MD ${ }^{1}$ | Maria Caterina Fortuna MD $^{1} \mid$ Gemma Caro MD $^{1}$ | Giulia Pranteda \\ MD $^{1}$ | Valentina Garelli MD ${ }^{1}$ | Umberto Pompili PhD ${ }^{1}$ | Marta Carlesimo MD ${ }^{2}$
}

${ }^{1}$ Department of Internal Medicine and Medical Specialties, "Sapienza" University of Rome, Rome, Italy

${ }^{2}$ Dermatology, "Sapienza" University of Rome, II School, Rome, Italy

Correspondence

Gemma Caro, Section of Dermatology, Department of Internal Medicine and Medical Specialties, University La Sapienza, Rome, Viale del Policlinico, 155 00161, Rome, Italy.

Email: gemmacaro90@gmail.com

\begin{abstract}
Summary
Background: Chemotherapy-induced alopecia (CIA) is probably one of the most shocking aspects for oncological patients and underestimated by physicians. Among hair loss risk factors, there are treatment-related aspects such as drug dose, administration regimen, and exposure to $\mathrm{X}$-rays, but also patient-related characteristics. To the best of our knowledge, no guidelines are available about CIA management.

Aims and methods: With this study, based on literature background and our clinical experience, we would like to propose a list of actions in order to estimate the risk of hair loss before starting chemotherapy and to manage this condition before, during, and after drug administration and to create a sort of practical guide for dermatologists and oncologists.

Results and conclusion: There is an urgent need for prospective studies to clarify the mechanistic basis of alopecia associated with these drugs and consequently to design evidence-based management strategies.
\end{abstract}

KEYWORDS

chemotherapeutic drugs, hair loss, skin reactions

\section{1 | BACKGROUND}

Chemotherapy-induced alopecia $(\mathrm{CIA})$ is probably one of the most shocking aspects for oncological patients and underestimated by physicians. It negatively influences body image, sexuality, and selfesteem, so that up to $8 \%$ of patients decide to refuse chemotherapy if there is the risk of hair loss. ${ }^{1}$

Its incidence is estimated to be $65 \%$, even if the prevalence and severity of hair loss are related to the drug category and to the number of the administered drugs. With antimicrotubule agents, it is reported more than $80 \%$ incidence of alopecia, $60 \%-100 \%$ with topoisomerase inhibitors, $>60 \%$ with alkylators, and 10\%-50\% with antimetabolites (Table 1). Polychemotherapy is associated with higher incidences compared to monotherapy. ${ }^{2}$

Among hair loss risk factors, there are treatment-related aspects such as drug dose, administration regimen, and exposure to $\mathrm{X}$-rays, but also patient-related features such as age, comorbidities, the presence of any type of alopecia (more frequently androgenetic alopecia), and nutritional and hormonal status. ${ }^{3}$

About hair-shaft shedding, it can be reached out from days to weeks after starting chemotherapy, and different shedding patterns can be observed (dystrophic anagen effluvium and telogen effluvium). Frontal or occipital hairlines are more frequently involved, suggesting that the affected areas seem to be selective.

Among factors that may influence the shedding pattern, the mitotic activity of the hair follicle in the moment of the insult is probably the more important. ${ }^{2}$ The main targets of anticancer drugs are the matrix keratinocytes, which are highly proliferative during anagen phase, and their pigmentary system. These structures are very sensitive to toxins and drugs, which can easily lead them to rapid apoptosis. ${ }^{2,3}$ Catagen and telogen are not affected because they are mitotically inactive phases, but when hair is in late anagen phase, characterized by a lower mitotic rate, chemotherapy accelerates the normal transition to telogen. ${ }^{2,3}$ 
TABLE 1 Incidence of alopecia associated with the main chemotherapeutic drug categories

\begin{tabular}{ll}
\hline Drug category & Incidence of alopecia \\
\hline Antimicrotubule agents & $80 \%$ \\
\hline Topoisomerase inhibitors & $60 \%-100 \%$ \\
\hline Alkylators & $>60 \%$ \\
\hline Antimetabolites & $10 \%-50 \%$ \\
\hline
\end{tabular}

If we consider that up to $90 \%$ of scalp hairs are normally anagen phase, it is easy to explain why the scalp is the most frequent affected area, whereas hairs of the beard, eyebrows, and eyelashes, as well as axillary and pubic regions, are affected depending on the percentage of hairs in anagen.

This kind of alopecia is generally reversible and hair usually regrows after 3-6 months, even if temporarily the new hair may show changes in color and/or texture. Although rare, cases of permanent alopecia, with severely retarded or without hair regrowth, are reported to be associated with high-dose chemotherapy or with busulfan and cyclophosphamide administration, and it is probably the consequence of a damage to hair follicle stem cells. ${ }^{2-4}$

We consider it is worthwhile to mention alopecia associated with aromatase inhibitors. The agents, such as letrozole and anastrozole, are associated with a recession of the frontal and parietal hairlines, diffuse hair loss, and miniaturization of follicles in the frontotemporal area, mimicking a typically female androgenetic alopecia (FAGA) with male pattern. The pathogenic mechanism of this type of hair loss seems to be associated with the decrease in estrogen synthesis. Estrogens are potent hair growth modulators and hair protective factors and derive from the conversion of androstenedione to estrone and testosterone to estradiol by aromatase (P450arom), the levels of which in frontal and occipital follicles of women are higher than in the same areas of men. The block of this enzyme induces a relative enhancement in the activity of $5 \alpha$-reductase which leads to a relative increase in amount of testosterone available for conversion to DHT. ${ }^{5}$

To the best of our knowledge, no guidelines are available about CIA management. The aim of this study was to propose a list of actions in order to estimate the risk of hair loss before starting chemotherapy and to manage this condition before, during, and after drug administration and to create a sort of guidelines for dermatologists and oncologists.

\section{2 | PRECHEMOTHERAPY MANAGEMENT}

Nowadays, no guidelines are available about prechemotherapy management. To estimate the risk of hair loss in a single patient before starting the treatment, it could be interesting to create a guide for dermatologists to analyze scalp condition. In our opinion, the following aspects should be evaluated.

First of all, patient selection is fundamental. Considering the better known therapeutic protocols and so the major knowledge about their side effects, in this study we considered only patients with solid tumor and not patients with hematological neoplasia.

Anamnestic data should be collected: Previous scalp diseases (psoriasis, seborrheic dermatitis, and atopic dermatitis, among the most frequent) and autoimmune diseases or a family history of alopecia could reveal a tendency to some types of alopecia or a subclinical disease that could become evident after chemotherapy. Obviously the drug protocol that will be administered is crucial to estimate the risk of hair loss.

In addition, some blood examinations could be requested. As is known, blood levels of hemoglobin and iron, thyroid hormones, and vitamin $D$ could be associated with hair loss, ${ }^{6-9}$ so that it may be desired to have sufficient blood levels of these substances before starting any type of anticancer drugs. ${ }^{10}$

Scalp dermoscopy (trichoscopy) is another rapid and noninvasive method that may help to unveil several scalp diseases or some types of alopecia that could be present even before chemotherapy. ${ }^{11}$ Trichoscopy can reveal an early stage of androgenetic alopecia, an alopecia areata incognita, or some kind of cicatricial alopecia such as a lichen planopilaris or a discoid lupus erythematosus that can lead to a permanent alopecia even without chemotherapy, but that could be accelerated and could get worse by anticancer drugs. ${ }^{12}$

Pull test and trichogram together with trichoscopy could also be a useful tool to evaluate hair condition at TO and to know whether pathological mechanisms are already acting. Moreover, trichogram could show hair cycle phase and therefore hair's susceptibility to chemotherapeutic agent damage.

All these evaluations can be considered to estimate the risk of hair loss after chemotherapy and to better manage alopecia during and after drug administration.

The following steps should be the prescription of drugs that could prevent hair loss. Even if no drugs are actually approved for this goal, some molecules have been studied.

Soref et al. ${ }^{13}$ studied the role of topical epinephrine or norepinephrine in preventing radiotherapy- and chemotherapyinduced alopecia in 10-day-old rats with good results and no side effects. This action of vasoconstrictors could probably be explained by a work of Rathman-Josserand et al. ${ }^{14}$ that hypothesized that the induction of hypoxia signaling could help hair follicle stem cells to maintain their function and hence prevent alopecia or at least favor neogenesis; moreover, vasoconstriction may reduce the drug dose that reaches the hair follicle, just as scalp cooling acts. When compared to scalp cooling, vasoconstrictors have the advantage of an easier administration, so that they can be applied more than once a day even before starting chemotherapy, ensuring both a preventing and a long-lasting effect; meanwhile, scalp cooling just acts during drug infusion.

On the basis of this experience, we are trying to use a galenic hydrogel, composed of ephedrine $0.5 \%$ and naphazoline $0.125 \%$, in our patients before the beginning of chemotherapy. We think that the use of these kinds of vasoconstrictors could be a useful tool to 
prevent chemo-induced alopecia, even if more studies are necessary.

\section{3 | DURING-CHEMOTHERAPY MANAGEMENT}

Implementation of gentle hair care strategies should be adopted throughout and after chemotherapy. A soft brush should be used, to avoid additional traumas, and hair should be washed only as often as necessary using a gentle shampoo. Cutting hair short or shaving hair is not necessary, but it could be more comfortable.

To help patients to deal with this condition and to protect the scalp from sun and cold exposure can be used a wig. To improve patients' psychological condition and allow them to use their own hair, an analogous wig could be used, obtained by cutting patients' hair and implanting them on a prosthetic support. ${ }^{2}$

Another instrument proposed as a preventive therapy for alopecia is scalp cooling. Actually, its mechanism is not well known, but it probably works through vasoconstriction, decreasing local concentration of chemotherapy and cellular uptake at the hair follicle, and reducing metabolic uptake. A recent meta-analysis reported that this method significantly reduces $\mathrm{CIA}^{15}$ and patients' compliance is good, even if some cases of headache and uncomfortable feelings could be associated ${ }^{1-3,16-18}$ as reported by Betticher et al. ${ }^{17}$ Best results have been described for alopecia induced by doxorubicin, epirubicin, and docetaxel, while it should be avoided in hematological malignancies because of the risk of scalp skin metastasis. ${ }^{2,3}$

Many drugs have been used to avoid chemo-induced alopecia, but no one has been approved.

In our opinion, the combination of topical steroids and topical vasoconstrictors could be a useful tool to prevent a massive damage to hair follicle, and in particular to hair follicle stem cells, with the goal to prevent a permanent alopecia.

\section{4 | POSTCHEMOTHERAPY MANAGEMENT}

To accelerate the regrowth after chemotherapy, $2 \%$ topical minoxidil is the best treatment. Its mechanism of action is not already known, but several hypotheses have been proposed, such as opening of potassium channels by its sulphated metabolite, stimulation of keratinocyte proliferation, inhibition of collagen synthesis and production, and stimulation of vascular endothelial growth factor (VEGF) and prostaglandin synthesis. It was demonstrated that minoxidil prolongs the duration of anagen and enlarges miniaturized follicles. ${ }^{19}$

It must be applied twice a day on the involved areas for at least 6 months. It has been demonstrated to reduce the severity and duration of alopecia. ${ }^{1-3}$

Also antioxidant agents could have a role in the postchemotherapy period. $\mathrm{N}$-acetylcysteine, an analog and precursor of glutathione, has been demonstrated to be capable of protecting neonatal rats from cyclophosphamide $\mathrm{CIA}$ and adult mice from doxorubicin $\mathrm{CIA}$ both when administered topically and parentally. ${ }^{20,21}$ Speaking of which it is necessary to pay attention because recently it has been reported that some kind of tumor may benefit from the reduction of oxidative stress caused by antioxidant, and this effect is obviously more important when they are parentally administered.

Another agent that has been used to facilitate hair regrowth after chemotherapy is calcitriol (1,25-dihydroxyvitamin D3), which was considered the most promising agent for treating $\mathrm{CIA}^{22,23}$ It has multiple effects on keratinocytes: inhibition of DNA synthesis, which causes cell cycle arrest at the G0/G1 interphase, inducing differentiation; inhibition of Ki67 expression, a marker of cycling cells; and inhibition of the growth of multiple other cell types. ${ }^{10,22-31}$ Hidalgo et al. ${ }^{32}$ also referred to contact dermatitis associated with 1,25-dihydroxyvitamin D3, and therefore, it must be used with caution in particular if applied topically.

\section{5 | TARGET THERAPY-INDUCED ALOPECIA}

What is exposed before is related to "classic" anticancer drugs that are better known. But several molecularly targeted anticancer therapies have been developed, with an impressive clinical benefit in terms of efficacy and survival rates, but also with new or different side effects. These new drugs may also cause alopecia, even if its characteristics are different from which we described for conventional chemotherapies. Belum reported an overall incidence of $14.7 \%$ of alopecia associated with these drugs (lowest for bortezomib and highest for vismodegib). ${ }^{33}$

In contrast to conventional chemotherapy, where the mechanism is predominantly nonselective cytotoxicity, the mechanisms at the basis of hair loss induced by these drugs are poorly understood. ${ }^{3}$ The primary target inhibited, type of the drug, variations in the target spectrum of inhibition, molecular cross talk between pathways, and the inherent role of these molecules in hair follicle biology may all be involved in the pathogenesis of hair loss.

These drugs selectively target various oncogenic molecules/pathways such as SMO, VEGFR, and MAPK, but the incidence of alopecia varies even among drugs acting on the same primary molecular target. Various pathogenic mechanisms may be involved considering two aspects: First, each one of these drugs often targets multiple other pathways, and second, the spectrum of inhibition and receptor affinity might vary from a drug to another. ${ }^{33}$

Concerning Shh pathway in hair follicle biology and epidermal homeostasis, murine studies have shown that its inhibition in the skin can lead to reversible alopecia and arrest of hair growth in the telogen phase. ${ }^{34}$ This explains vismodegib-induced alopecia.

Inhibition of the EGFR is associated with follicular disintegration accompanied by inflammation because of its crucial role in anagencatagen transition. ${ }^{35-37}$

The onset and pattern of alopecia are not well described. The alopecia may be frontal (androgenetic-like), diffuse, or patchy, and it is generally nonscarring ${ }^{38,39}$; pruritus may also be present. In some 
cases, scarring alopecia/folliculitis decalvans with pain and infection may develop. ${ }^{40,41}$

The onset after the beginning of treatment may range from 2 weeks to months and resolve within 1-6 months after drug discontinuation; the quality of hair and rate of regrowth may also be affected. $^{42}$

With the expanding use of targeted agents, there is an urgent need for prospective studies to clarify the mechanistic basis of alopecia associated with these drugs and consequently to design evidence-based management strategies.

Moreover, these conditions should be treated among several specialists, to reduce evaluation times and simplify their management. The dermatologist has a crucial role in every aspect of the treatment phase and should work together with the oncologist in taking care of these patients.

\section{CONFLICT OF INTEREST}

All the authors disclose no financial, consulting, and personal relationships with other people or organizations that could influence (bias) the authors' work. The authors also disclose no scientific writing assistance, grant support and numbers (including NIH/Wellcomefunded papers), and statements of employment.

\section{REFERENCES}

1. Balagula Y, Rosen ST, Lacouture ME. The emergence of supportive oncodermatology: the study of dermatologic adverse events to cancer therapies. J Am Acad Dermatol. 2011;65:624-635.

2. Trüeb RM. Chemotherapy-induced hair loss. Skin Therapy Lett 2010;15:5-7.

3. Paus R, Haslam IS, Sharov AA, Botchkarev VA. Pathobiology of chemotherapy-induced hair loss. Lancet Oncol. 2013;14:e50-e59.

4. Miteva M, Misciali C, Fanti PA, Vincenzi C, Romanelli P, Tosti A. Permanent alopecia after systemic chemotherapy: a clinicopathological study of 10 cases. Am J Dermatopathol. 2011;33:345-350.

5. Rossi A, lorio A, Scali E, et al. Aromatase inhibitors induce 'male pattern hair loss' in women? Ann Oncol. 2013;24:1710-1711.

6. Park SY, Na SY, Kim JH, Cho S, Lee JH. Iron plays a certain role in patterned hair loss. J Korean Med Sci. 2013:28:934-938.

7. Trüeb RM. Hormones and hair growth. Hautarzt. 2010;61:487-495.

8. Reichrath J, Schilli M, Kerber A, Bahmer FA, Czarnetzki BM, Paus R. Hair follicle expression of 1,25-dihydroxyvitamin D3 receptors during the murine hair cycle. Br J Dermatol. 1994;131:477-482.

9. Aoi N, Inoue K, Chikanishi T, et al. 1,25-dihydroxyvitamin D3 modulates the hair-inductive capacity of dermal papilla cells: therapeutic potential for hair regeneration. Stem Cells Transl Med. 2012;1:615 626.

10. Jimenez JJ, Yunis AA. Protection from chemotherapy-induced alopecia 1,25-dihydroxyvitamin D3. Cancer Res. 1992;52:5123-5125.

11. Rudnicka L, Olszewska M, Rakowska A, Kowalska-Oledzka E, Slowinska M. Trichoscopy: a new method for diagnosing hair loss. J Drugs Dermatol. 2008;7:651-654.

12. Miteva M, Tosti A. Hair and scalp dermatoscopy. J Am Acad Dermatol. 2012;67:1040-1048

13. Soref CM, Fahl WE. A new strategy to prevent chemotherapy and radiotherapy-induced alopecia using topically applied vasoconstrictor. Int J Cancer. 2015;136:195-203.
14. Rathman-Josserand M, Genty G, Lecardonnel J, et al. Human hair follicle stem/progenitor cells express hypoxia markers. J Invest Dermatol. 2013;133:2094-2097.

15. Hyoseung S, Seong JJ, Do HK, Ohsang K, Seung-K M. Efficacy of interventions for prevention of chemotherapy-induced alopecia: a systematic review and meta-analysis. Int J Cancer. 2015;136:E442-E454.

16. Galimont-Collen AF, Vos LE, Lavrijsen AP, Ouwerkerk J, Gelderblom $\mathrm{H}$. Classification and management of skin, hair, nail and mucosal side-effects of epidermal growth factor receptor (EGFR) inhibitors. Eur J Cancer. 2007;43:845-851.

17. Betticher DC, Delmore G, Breitenstein U, et al. Efficacy and tolerability of two scalp cooling systems for the prevention of alopecia associated with docetaxel treatment. Support Care Cancer. 2013;21:2565-2573.

18. Shin H, Jo SJ, Kim DH, Kwon O, Myung SK. Efficacy of interventions for prevention of chemotherapy-induced alopecia: a systematic review and meta-analysis. Int J Cancer. 2015;136:E442-E454.

19. Messenger AG, Rundegren J. Minoxidil: mechanisms of action on hair growth. Br J Dermatol. 2004;150:186-194.

20. Agostini FD, Bagnasco M, Giunciuglio D, Albini A, De Flora S. Inhibition by oral $\mathrm{N}$-acetylcysteine of doxorubicin-induced clastogenicity and alopecia, and prevention of primary tumors and lung micrometastases in mice. Int J Oncol. 1998;13:217-224.

21. Jimenez JJ, Haung HS, Yunis AA. Treatment with ImuVert/N-acetylcysteine protects rats from cyclophosphamide/cytarabine-induced alopecia. Cancer Invest. 1992;10:271-276.

22. Jimenez JJ, Yunis AA. Vitamin D3 and chemotherapy-induced alopecia. Nutrition. 1996;12:448-449.

23. Wang J, Lu Z, Au JL. Protection against chemotherapy-induced alopecia. Pharm Res. 2006;23:2505-2514.

24. Kobayashi T, Hashimoto K, Yoshikawa K. Growth inhibition of human keratinocytes by 1,25-dihydroxyvitamin D3 is linked to dephosphorylation of retinoblastoma gene product. Biochem Biophys Res Commun. 1993:196:487-493.

25. Kobayashi T, Okumura H, Hashimoto K, Asada H, Inui S, Yoshikawa K. Synchronization of normal human keratinocyte in culture: its application to the analysis of 1,25-dihydroxyvitamin D3 effects on cell cycle. J Dermatol Sci. 1998;17:108-114.

26. Blutt SE, Allegretto EA, Pike JW, Weigel NL. 1,25-dihydroxyvitamin D3 and 9-cis-retinoic acid act synergistically to inhibit the growth of LNCaP prostate cells and cause accumulation of cells in G1. Endocrinology. 1997;138:1491-1497.

27. Hager G, Formanek M, Gedlicka C, Thurnher D, Knerer B, Kornfeh J. 1,25(OH)2 vitamin D3 induces elevated expression of the cell cycle-regulating genes P21 and P27 in squamous carcinoma cell lines of the head and neck. Acta Otolaryngol. 2001;121:103-109.

28. Kawa S, Yoshizawa K, Tokoo M, et al. Inhibitory effect of 220-oxa1,25-dihydroxyvitamin D3 on the proliferation of pancreatic cancer cell lines. Gastroenterology. 1996;110:1605-1613.

29. Kornfehl J, Formanek M, Temmel A, Knerer B, Willheim M. Antiproliferative effects of the biologically active metabolite of vitamin D3 $(1,25[\mathrm{OH}] 2 \mathrm{D} 3)$ on head and neck squamous cell carcinoma cell lines. Eur Arch Otorhinolaryngol. 1996;253:341-344.

30. Liu M, Lee MH, Cohen M, Bommakanti M, Freedman LP. Transcriptional activation of the Cdk inhibitor p21 by vitamin D3 leads to the induced differentiation of the myelomonocytic cell line U937. Genes Dev. 1996;10:142-153.

31. Jimenez JJ, Alvarez E, Bustamante CD, Yunis AA. Pretreatment with 1,25(OH)2D3 protects from Cytoxan-induced alopecia without protecting the leukemic cells from Cytoxan. Am J Med Sci. 1995;310:43-47.

32. Hidalgo M, Rinaldi D, Medina G, Griffin T, Turner J, Von Hoff DD. A phase I trial of topical topitriol (calcitriol, 1,25-dihydroxyvitamin D-3) to prevent chemotherapy-induced alopecia. Anticancer Drugs. 1999;10:393-395. 
33. Belum VR, Marulanda K, Ensslin C, et al. Alopecia in patients treated with molecularly targeted anticancer therapies. Ann Oncol. 2015;26:2496-2502.

34. Nanney LB, Magid M, Stoscheck CM, King LE Jr. Comparison of epidermal growth factor binding and receptor distribution in normal human epidermis and epidermal appendages. J Invest Dermatol. 1984;83:385-393.

35. Philpott MP, Kealey T. Effects of EGF on the morphology and patterns of DNA synthesis in isolated human hair follicles. J Invest Dermatol. 1994;102:186-191.

36. Hansen LA, Alexander N, Hogan ME, et al. Genetically null mice reveal a central role for epidermal growth factor receptor in the differentiation of the hair follicle and normal hair development. Am J Pathol. 1997;150:1959-1975.

37. Lacouture ME. Mechanisms of cutaneous toxicities to EGFR inhibitors. Nat Rev Cancer. 2006;6:803-812.

38. Robert C, Mateus C, Spatz A, et al. Dermatologic symptoms associated with the multikinase inhibitor sorafenib. J Am Acad Dermatol. 2009;60:299-305.

39. Osio A, Mateus C, Soria JC, et al. Cutaneous side-effects in patients on long-term treatment with epidermal growth factor receptor inhibitors. Br J Dermatol. 2009;161:515-521.
40. Hepper DM, Wu P, Anadkat MJ. Scarring alopecia associated with the epidermal growth factor receptor inhibitor erlotinib. J Am Acad Dermatol. 2011;64:996-998.

41. Hoekzema R, Drillenburg P. Folliculitis decalvans associated with erlotinib. Clin Exp Dermatol. 2010;35:916-918.

42. Lacouture ME, Anadkat MJ, Bensadoun RJ, et al. Clinical practice guidelines for the prevention and treatment of EGFR inhibitor-associated dermatologic toxicities. Support Care Cancer. 2011;19:10791095.

How to cite this article: Rossi A, Fortuna MC, Caro G, et al. Chemotherapy-induced alopecia management: Clinical experience and practical advice. J Cosmet Dermatol. 2017;00:1-6. doi:10.1111/jocd.12308. 